\title{
The Impact of Sham Feeding with Chewing Gum on Postoperative lleus Following Colorectal Surgery: a Meta-Analysis of Randomised Controlled Trials
}

\author{
Farah Roslan ${ }^{1,2} \cdot$ Anisa Kushairi ${ }^{1,2} \cdot$ Laura Cappuyns $^{1,2} \cdot$ Prita Daliya $^{1,2} \cdot$ Alfred Adiamah $^{1,2}$ (I) \\ Received: 6 August 2019 / Accepted: 16 December 2019 / Published online: 26 February 2020 \\ (C) 2020 The Author(s)
}

\begin{abstract}
Background Chewing gum as a form of sham feeding is an inexpensive and well-tolerated means of promoting gastrointestinal motility following major abdominal surgery. Although recognised by the Enhanced Recovery After Surgery (ERAS) Society as one of the multimodal approaches to expedite recovery after surgery, strong evidence to support its use in routine postoperative practice is lacking.

Methodology A comprehensive literature review of all randomised controlled trials (RCTs) was performed in the Medline and Embase databases between 2000 and 2019. Studies were selected to compare the use of chewing gum versus standard care in the management of postoperative ileus (POI) in adults undergoing colorectal surgery. The primary outcome assessed was the incidence of POI. Secondary outcomes included time to passage of flatus, time to defecation, total length of hospital stay and mortality.

Results Sixteen RCTs were included in the systematic review, of which ten (970 patients) were included in the meta-analysis. The incidence of POI was significantly reduced in patients utilising chewing gum compared to those having standard care (RR 0.55, $95 \% \mathrm{CI} 0.39,0.79, p=0.0009$ ). These patients also had a significant reduction in time to passage of flatus (WMD $-0.31,95 \% \mathrm{CI}$ $-0.36,-0.26, p<0.00001$ ) and time to defecation (WMD $-0.47,95 \% \mathrm{CI}-0.60,-0.34, p<0.00001$ ), without significant differences in the total length of hospital stay or mortality.

Conclusion The use of chewing gum after colorectal surgery is a safe and effective intervention in reducing the incidence of POI and merits routine use alongside other ERAS pathways in the postoperative setting.
\end{abstract}

Keywords Sham feeding $\cdot$ Chewing gum $\cdot$ Postoperative ileus $\cdot$ Colorectal surgery

This paper was presented at the Society of Academic and Research Surgery and Royal Society of Medicine 2019 meeting. The meeting abstract was published in the British Journal of Surgery (https://doi.org/ 10.1002/bjs.2019.106.issue-S3)

Farah Roslan and Anisa Kushairi are joint first authors.

Alfred Adiamah

Alfred.adiamah@nuh.nhs.uk

1 Gastrointestinal Surgery, Nottingham Digestive Diseases Centre and National Institute for Health Research (NIHR) Nottingham Biomedical Research Centre, Nottingham University Hospitals NHS Trust and University of Nottingham, Queen's Medical Centre Campus, Derby Road, Nottingham NG7 2UH, UK

2 East Midlands Surgical Academic Network (EMSAN), Queen's Medical Centre Campus, Derby Road, Nottingham NG7 2UH, UK

\section{Introduction}

Postoperative ileus (POI) is the temporary inhibition of gastrointestinal motility due to non-mechanical causes. Occurring after abdominal surgery, particularly after handling the bowel, it may result in nausea, vomiting and anorexia. ${ }^{1}$ Despite the lack of standard clinical definitions, the incidence of POI is reported to occur in up to 1 in 4 patients who have undergone gastrointestinal surgery making POI arguably the most frequent complication following digestive surgery. ${ }^{2,}{ }^{3}$ Its sequelae include malnutrition, dehydration, electrolyte imbalance and aspiration pneumonia. POI poses a significant socioeconomic impact by prolonging hospital stay by as much as 5 days per patient and costing a staggering sum of 1.46 billion USD per annum to the health economy. ${ }^{3,4}$ Surprisingly, not much progress has been made over the years in reducing its incidence and consequences. 


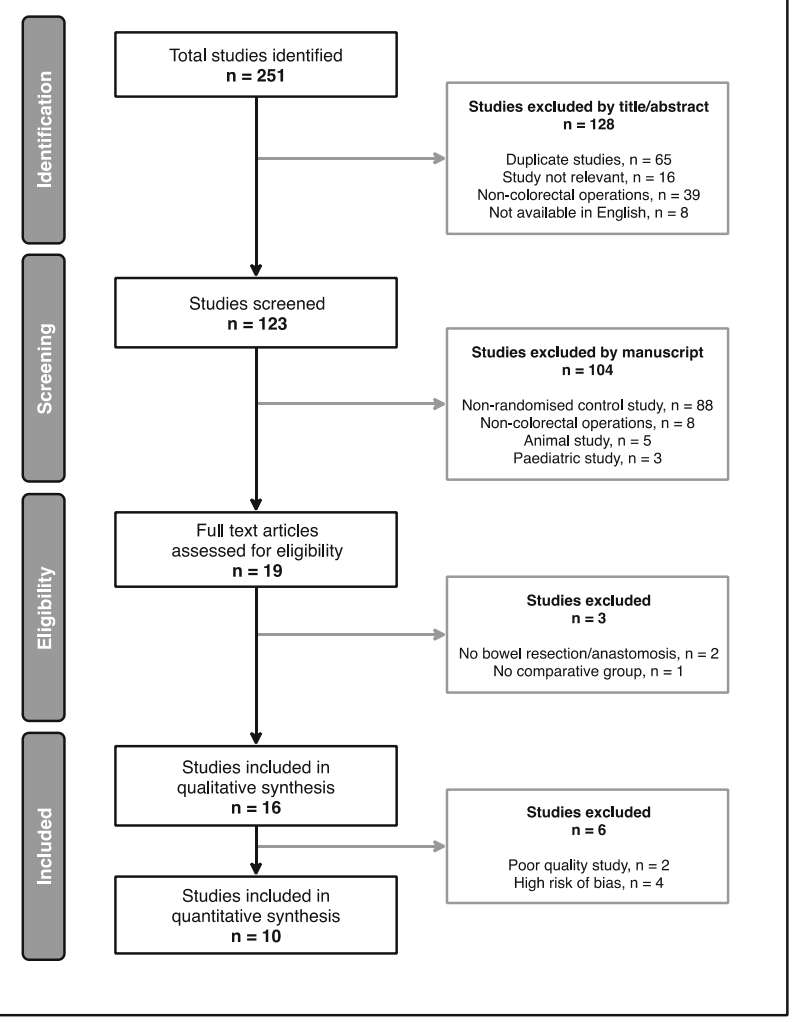

Fig. 1 Preferred Reporting Items for Systematic reviews and MetaAnalysis (PRISMA) flow diagram

The underlying mechanism of POI remains elusive; postulated as ensuing from surgical manipulation, intestinal oedema, electrolyte imbalance and medication such as opioids. ${ }^{5}$ The avoidance and management of risk factors can best be realised by application of multimodal pathways incorporating minimally invasive surgery, a stringent fluid regimen, the use of modern opioid-sparing pain strategies and early mobilisation. ${ }^{5,6}$

Chewing gum, alongside other multipronged approaches recognised by the Enhanced Recovery After Surgery (ERAS) Society, ${ }^{7}$ is thought to simulate sham feeding which may stimulate gastrointestinal recovery postoperatively by the activation of the cephalic-vagal axis. ${ }^{6,8,9}$ This approach in sham feeding, is thought to encourage or initiate the processes involved in gut recovery without actually challenging the gut with food. Although early enteral feeding is recommended and widely practiced in the era of enhanced recovery, an estimated $20 \%$ of patients are unable to tolerate oral intake after the first postoperative day. ${ }^{10,11}$ Additionally, many practitioners are reluctant to institute early feeding in some cases due to fears over safety and potential complications. ${ }^{12}$ Sham feeding could therefore be considered a safer alternative to early enteral nutrition in reducing POI.

Despite ERAS Society guidelines recommending the use of postoperative chewing gum to reduce POI, existing literature including a 2015 Cochrane review ${ }^{13}$ have been

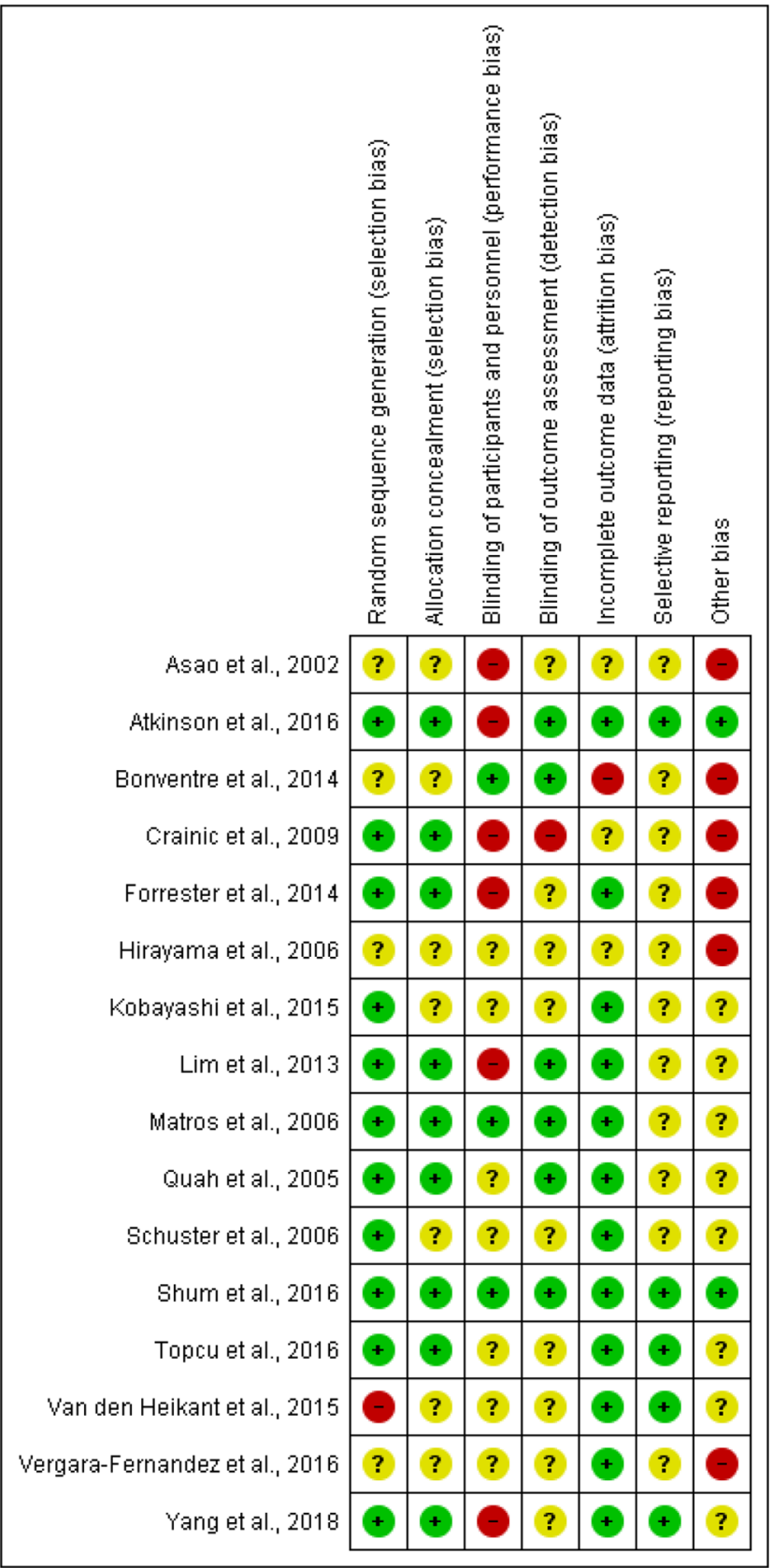

Fig. 2 Summary of risk of bias assessment of the included studies

inconclusive in providing sufficient evidence for its use. Poor quality trials with small patient numbers, variation in the definition of POI, diverse perioperative care settings and heterogeneity in the operative procedures studied may all be contributing to the inconsistent evidence seen.

The aim of this meta-analysis is, therefore, to provide a valid and up-to-date summary of relevant high-quality trials comparing the impact of chewing-gum compared to standard care (the use of controls or placebos) in the management of $\mathrm{POI}$ in adults undergoing resectional large bowel surgery with or without an anastomosis. The primary outcome assessed 


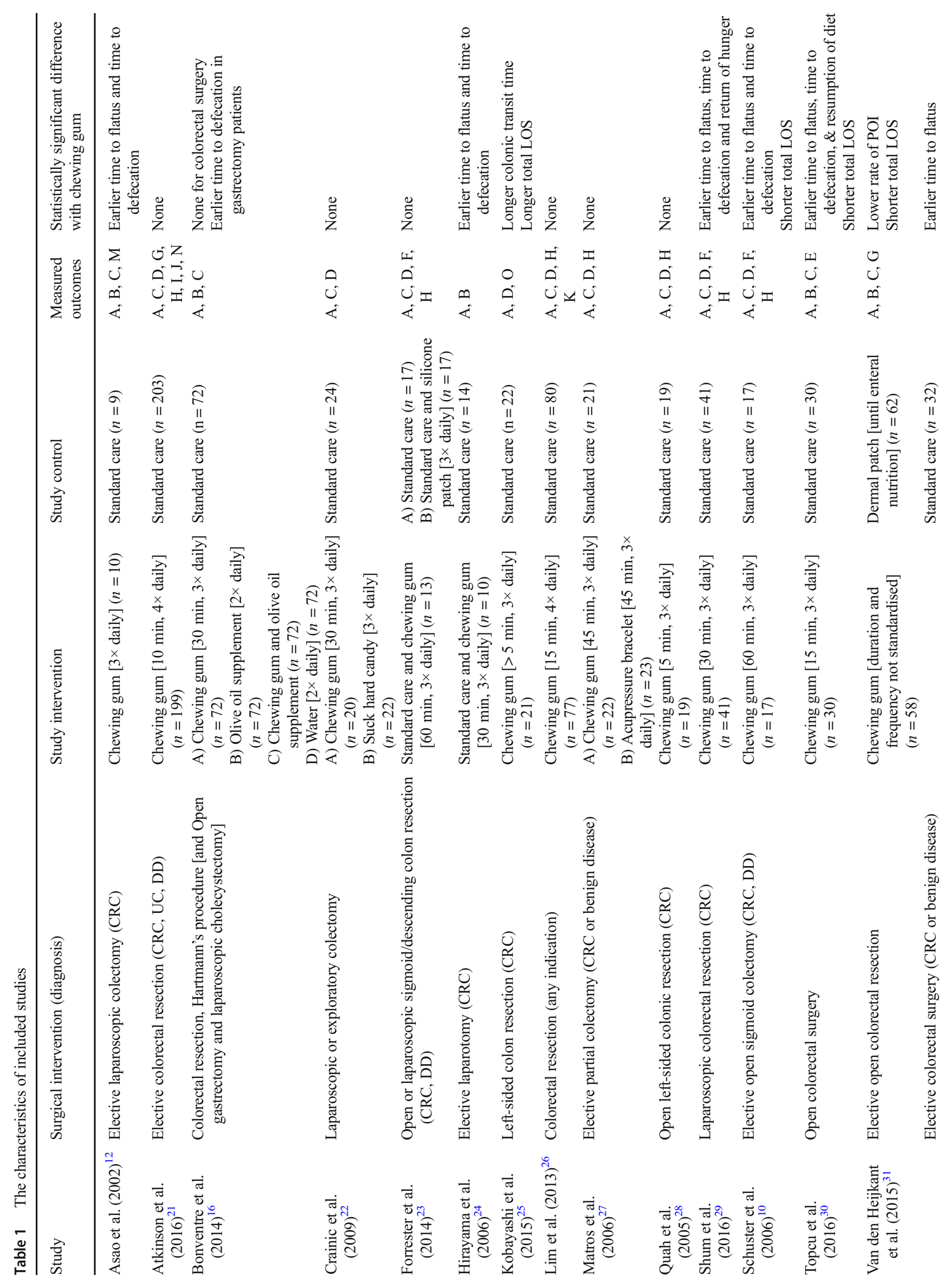


was the incidence of POI. Secondary outcomes included time to passage of flatus, time to defecation, total length of hospital stay and mortality.

\section{Methodology}

\section{Search Strategy}

A comprehensive literature review of the Medline and Embase databases was conducted between 2000 and 2019. Search criteria were used to identify all studies evaluating the effect of postoperative chewing gum versus either a control or placebo on POI in patients undergoing open or laparoscopic colorectal surgery. The electronic search terms used were ["chewing gum" OR "sham feeding"] AND ["colorectal surgery"] AND ["postoperative ileus" OR "paralytic ileus"]. The Scottish Intercollegiate Guidelines Network (SIGN) ${ }^{14}$ filter was used to restrict studies to randomised controlled trials (RCTs). Only studies published in English and those involving adult patients over 16 years of age were included. Hand searches of the bibliographies of all included studies were performed to ensure comprehensive study inclusion. The meta-analysis was conducted according to the Preferred Reporting Items for Systematic Reviews and Meta-Analyses (PRISMA) statement. ${ }^{15}$

\section{Selection of Article}

Preliminary exclusion was performed manually following title and abstract review (FR and $\mathrm{AK}$ ) and subsequently through full-text review (AK, FR and AA) as illustrated in Fig. 1. "Colorectal surgery" included open, laparoscopic, emergency or elective procedures where the bowel was handled and resected with or without an anastomosis. Studies which involved patients primarily undergoing day-case or noncolorectal operations (upper gastrointestinal, hepatobiliary, bariatric, gynaecological, urological or vascular) were excluded, as were studies which did not report on any relevant clinical outcome measures, and studies where both patient groups received chewing gum. All except one study ${ }^{16}$ which reported on outcomes following mixed gastrointestinal surgery were excluded; this study was retained as colorectal and noncolorectal data were reported separately. ${ }^{16}$

\section{Data Extraction}

Two independent authors (FR and AK) extracted data from the included studies according to predefined criteria. All RCTs were assessed for methodology, study design, inclusion and exclusion criteria, and outcome measures. Studies which presented their data as 'median and range' values were converted to 'means and standard deviation (SD)' data using the 


\begin{tabular}{|c|c|c|c|c|c|}
\hline \multirow[b]{2}{*}{ Study or Subgroup } & \multicolumn{2}{|c|}{ Chewing Gum } & \multicolumn{2}{|c|}{ Control } & \multirow[b]{2}{*}{ Weight } \\
\hline & Events & Total & Events & Total & \\
\hline Atkinson et al., 2016 & 19 & 199 & 28 & 202 & $40.6 \%$ \\
\hline Van den Heikant et al., 2015 & 14 & 52 & 29 & 60 & $45.5 \%$ \\
\hline Vergara-Femandez et al., 2016 & 2 & 32 & 7 & 32 & $5.5 \%$ \\
\hline Yang et al., 2018 & 3 & 43 & 11 & 46 & $8.4 \%$ \\
\hline Total $(95 \% \mathrm{Cl})$ & & 326 & & 340 & $100.0 \%$ \\
\hline Total events & 38 & & 75 & & \\
\hline
\end{tabular}

Fig. 3 Incidence of postoperative ileus

methods described by Hozo et al. and Wan et al. enabling all relevant data to be included in quantitative synthesis. ${ }^{17,} 18$

The methodological quality of each RCT was recorded for methods of randomisation, blinding, protocol violation and allocation concealment. All studies were scored using the Jadad scale. ${ }^{19}$ Any disagreement was resolved by consensus discussions with the senior member of the review team (AA). Data collected included type of surgery, execution of intervention and control measures, measured outcomes and statistically significant differences pertaining to chewing gum.

A risk of bias assessment was performed (PD, FR and AK) using the Cochrane Collaboration tool in RevMan $5.3,{ }^{20}$ which focuses upon random sequence generation (selection bias), allocation concealment (selection bias), blinding of participants and personnel (performance bias), blinding of outcome assessment (detection bias), incomplete outcome data (attrition bias) and selective reporting (reporting bias). Each study was ranked as low, moderate or high risk of bias based on these criteria (Fig. 2).

\section{Statistical Analysis}

Effect sizes for dichotomous outcomes were reported as risk ratio (RR) with 95\% confidence intervals (CIs) and as weighted mean difference (WMD) for continuous data. Only studies of low to moderate risk of bias were included in quantitative pooling. Given the variability in the operative interventions, the different approaches (open versus laparoscopic) and the timings of chewing gum administration, it was decided a priori that a random effects model would be most appropriate for this meta-analysis.

Small studies with no power calculation (participant numbers less than 20), or those with high risk of bias were excluded from quantitative analysis to avoid overstating or understating the treatment effects. Statistical heterogeneity was assessed by the $I^{2}$ statistic; threshold values of $I^{2}$ equal to $25 \%, 50 \%$ and $75 \%$ represent low, moderate and high heterogeneity, respectively. These analyses were performed using RevMan 5.3 software (The Nordic Cochrane Center, The Cochrane Collaboration, Copenhagen, Denmark). ${ }^{20}$

\section{Protocol Registration}

The study protocol for this meta-analysis was registered (CRD42018115852) with the PROSPERO database (www. crd.york.ac.uk/prospero).

\section{Results}

\section{Study Quality}

All selected studies included patients who underwent colorectal surgery. Table 1 summarises the characteristics of the sixteen included qualitative studies, ten of which were included in the quantitative synthesis. ${ }^{21,23,25-29,31-33}$ A total of 970 patients undergoing colorectal surgery were randomised to either postoperative chewing gum $(n=481)$ or routine postoperative care $(n=489)$.

\section{Qualitative Analysis}

Overall, the findings from the qualitative analyses were mixed and inconclusive. Qualitative analysis saw no significant difference in the incidence of POI between the chewing gum and control groups. ${ }^{12,26}$ Four studies saw no difference in time to passage of flatus in patients who had chewing gum and those in the control group, ${ }^{10}, 16,21,22$ while three studies found a significant reduction. ${ }^{12,24,30}$ Qualitative analysis of time to passage of stool saw no significant differences in three studies, ${ }^{16,22,23}$ while four studies showed significant reductions when using chewing gum. ${ }^{10,12,24,30}$ In terms of length of hospital stay, two studies showed no significant reduction, ${ }^{12,16}$ while two studies showed a significant reduction in length of stay. ${ }^{10,30}$

\section{Primary Outcome}

\section{Incidence of Postoperative lleus}

In total, meta-analysis of four studies revealed 326 patients in the chewing gum group and 340 patients in the control group who showed significantly reduced incidence of POI (RR 0.55 , $95 \%$ CI $0.39,0.79, p=0.0009, I^{2}=0 \%$ ) (Fig. 3$) .{ }^{21,31-33}$ Very 


\begin{tabular}{|c|c|c|c|c|c|c|c|}
\hline \multirow[b]{2}{*}{ Study or Subgroup } & \multicolumn{3}{|c|}{ Chewing Gum } & \multicolumn{3}{|c|}{ Control } & \multirow[b]{2}{*}{ Weight } \\
\hline & Mean & SD & Total & Mean & SD & Total & \\
\hline Forrester et al., 2014 & 2.98 & 3.16 & 13 & 3.42 & 2.18 & 17 & $0.1 \%$ \\
\hline Kobayashi et al., 2015 & 2.21 & 0.08 & 21 & 2.04 & 1.08 & 22 & $1.2 \%$ \\
\hline Lim et al., 2013 & 1.78 & 0.16 & 77 & 2.12 & 0.16 & 80 & $46.4 \%$ \\
\hline Matros et al., 2006 & 2.63 & 0.76 & 22 & 2.9 & 0.92 & 21 & $0.9 \%$ \\
\hline Quah et al., 2005 & 2.4 & 1 & 19 & 2.7 & 1 & 19 & $0.6 \%$ \\
\hline Shum et al., 2016 & 1.57 & 2.63 & 41 & 2.57 & 4.39 & 41 & $0.1 \%$ \\
\hline Yang et al., 2018 & 1.76 & 0.14 & 43 & 2.05 & 0.06 & 46 & $50.8 \%$ \\
\hline Total $(95 \% \mathrm{Cl})$ & & & 236 & & & 246 & $100.0 \%$ \\
\hline
\end{tabular}

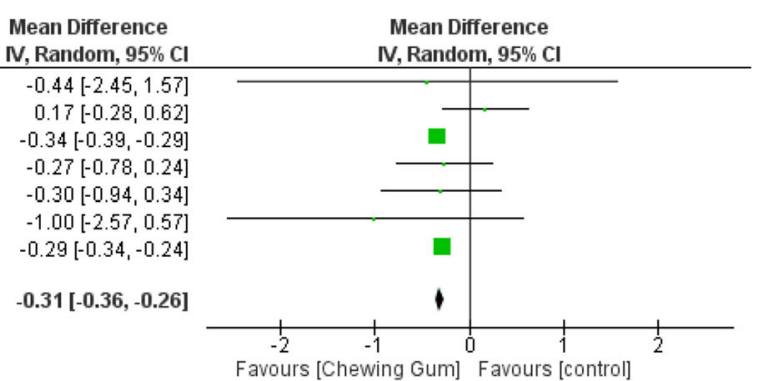

Fig. 4 Time to passage of flatus

low heterogeneity was observed in reporting the incidence of POI. The percentage of POI reported was lower in the gumchewing arm ranging from 6 to $27 \%$, whereas the control arm had a higher range between 14 and $48 \%$ (Table 3).

\section{Secondary Outcomes}

\section{Time to Passage of Flatus}

Seven RCTs (482 patients) were included in the quantitative meta-analysis of time to passage of flatus. Meta-analysis of 236 patients given chewing gum and 246 patients on placebo treatment showed significantly shorter time to passage of flatus in the chewing gum group (WMD $-0.31,95 \% \mathrm{CI}-0.36$, $-0.26, p<0.00001$ ) (Fig. 4). ${ }^{23,}{ }^{25-29,}{ }^{33}$ Heterogeneity was low with an $I^{2}$ value of $17 \%$.

\section{Time to Defecation}

Eight RCTs with 886 patients in total were included in the meta-analysis of time to defecation. Meta-analysis of 391 patients in the chewing gum group and 395 patients in the control group showed a significant decrease in time to defecation (data given in days) in the chewing gum group (WMD -0.47 , 95\% CI $-0.60,-0.34, p<0.00001$ ) (Fig. 5). ${ }^{21,23,25-29,33}$ Heterogeneity was moderate at $I^{2}$ of $55 \%$.

\section{Length of Hospital Stay}

Eight RCTs ( 823 patients) were included in the meta-analysis of length of hospital stay. Meta-analysis of 405 patients in the chewing gum group and 418 patients in the control group showed no significant reduction in length of stay (data presented in number of days) in the chewing gum group (WMD $-0.18,95 \%$ CI $-0.92,0.55, p=0.28, I^{2}=19 \%$ ) (Fig. 6). ${ }^{21,23 \text {, }}$ $25,27-29,31,32$

\section{Mortality}

Of the 16 included studies, five RCTs (780 patients) reported findings on patient mortality. ${ }^{21,26,28,31,32}$ Meta-analysis of 385 patients in the chewing gum group and 395 patients in the control group showed no significant difference in mortality between both groups (RR 2.10, 95\% CI 0.51, 8.76, $p=0.59$, $\left.I^{2}=0 \%\right)$ (Fig. 7).

\section{Discussion}

This systematic review of sixteen RCTs, of which ten were metaanalysed, comparing the efficacy of postoperative chewing gum against standard postoperative care demonstrated that chewing gum played a significant role in reducing the incidence of POI, time to passage of flatus and time to defecation in patients who had resectional large bowel surgery with or without an anastomosis. These findings were without any significant changes to total length of hospital stay or mortality (Fig. 8).

\section{Study Strengths}

By selecting RCTs involving only adult patients who had undergone colorectal surgery meant that this systematic

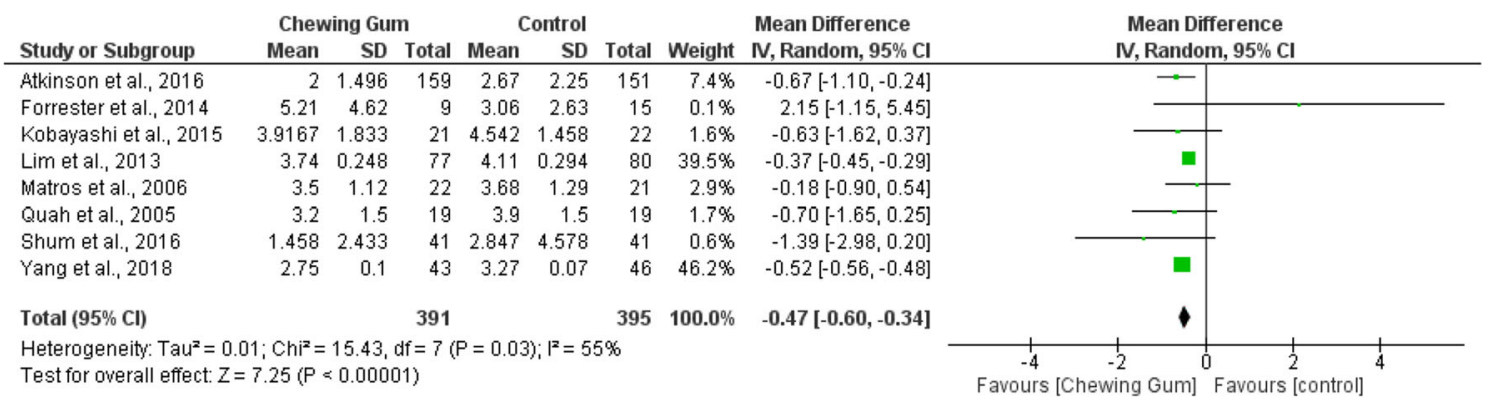

Fig. 5 Time to defecation 


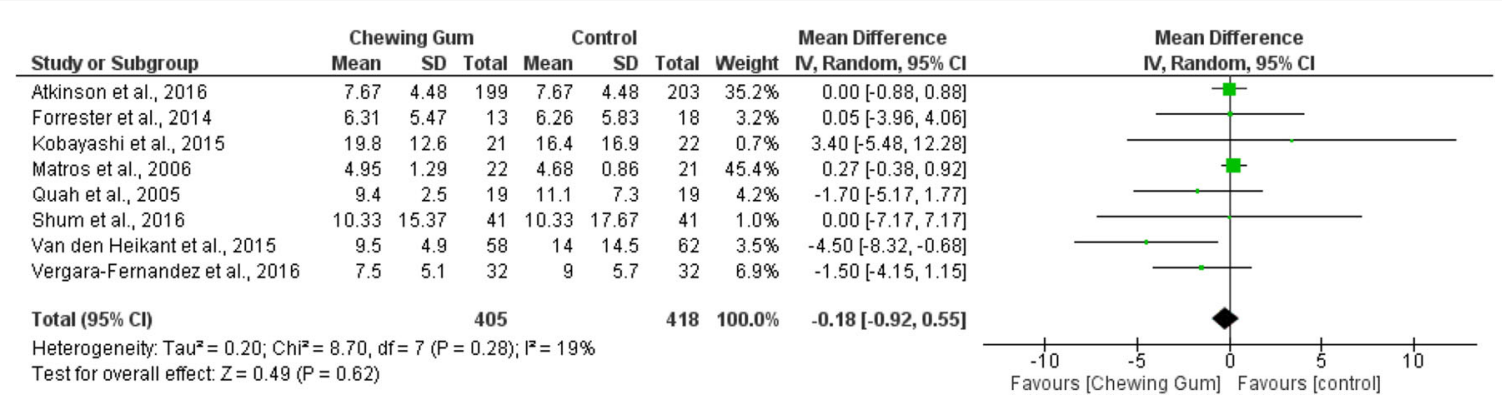

Fig. 6 Length of hospital stay

review and meta-analysis achieved a more homogenous group of patients in which to perform a quantitative analysis of outcomes. The removal of confounding factors and exclusion of patients with a smaller likelihood of ileus such as those undergoing day-case surgery also made the findings more relevant to the management of patients undergoing colorectal surgery.

In addition to outcomes explored in previous systematic reviews, we performed a meta-analysis on the incidence of POI and mortality. This paper is one of the few systematic reviews to directly explore the incidence of POI as the primary endpoint instead of the time to passage of flatus, which is the more commonly reported outcome in most RCTs. Our analysis demonstrated a significantly lower incidence of POI in patients who had chewing gum compared to patients who did not. In measuring the time to passage of flatus as the primary outcome, there is a potential for lack of accuracy in patient self-reporting and clinician documentation and this may explain the high heterogeneity and inconclusive findings reported in earlier meta-analyses.

Quality assessment revealed a wide variation between the included qualitative studies. A focus on moderate to high methodological quality studies, and those with a lower risk of bias was very important in formulating an accurate and relevant meta-analysis. In this review, we have managed to perform quantitative analysis of ten such relevant studies, which has resulted in a significant reduction of heterogeneity of the outcomes when compared to other meta-analyses on this topic.

\section{Limitations of Study}

The major confounders that may have had an influence on the study outcomes include the type and duration of colorectal operations, minor variations of the definition of POI, outcome reporting and the temporal spread of studies in relation to the implementation of ERAS. However, after looking into descriptions of what constituted POI in each of the included studies (Table 2), we found that the four studies which reported on incidence of POI had relatively similar definitions which would limit the heterogeneity and minimise the potential for reporting bias. ${ }^{21,31-33}$ This is also reflected in the significant and homogenous outcome of the primary endpoint.

Nevertheless, there were also significant sources of heterogeneity amongst the studies including clinical factors such as the increasing implementation of ERAS protocols, and the impact of other postoperative complications unrelated to POI. Other sources of heterogenicity which have been well documented but difficult to quantify from the studies selected were non-clinical factors such as social care and occupational therapy. ${ }^{34,35}$ These differences may be due to the geographical spread of the studies, or variation in operative management (Table 3).

There was a large variation in the implementation of the intervention, in particular the duration and frequency of chewing gum administration in each of the studies. This ranged from as short as $5 \mathrm{~min}$ of chewing 3 times daily, to as long as $60 \mathrm{~min}$ of chewing 3 times daily. One of the studies did not standardise the duration or frequency of gum chewing by allowing patients to chew gum to their own liking. ${ }^{31}$

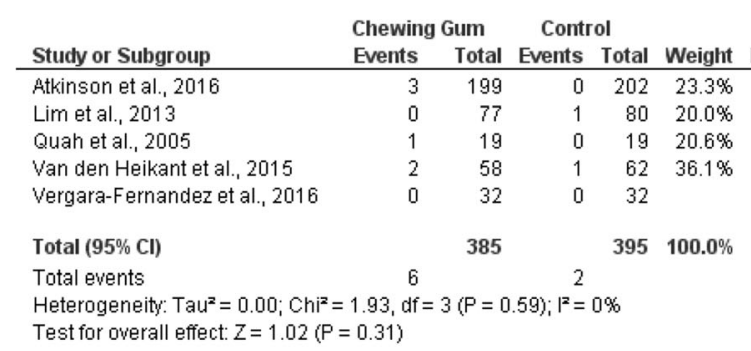

Risk Ratio

V, Random, $95 \% \mathrm{Cl}$ $7.11[0.37,136.67]$ $0.35[0.01,8.37]$ $3.00[0.13,69.31]$

$2.14[0.20,22.95]$

Not estimable

$2.10[0.51,8.76]$

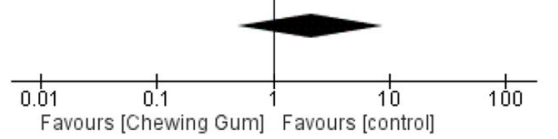

Fig. 7 Mortality 


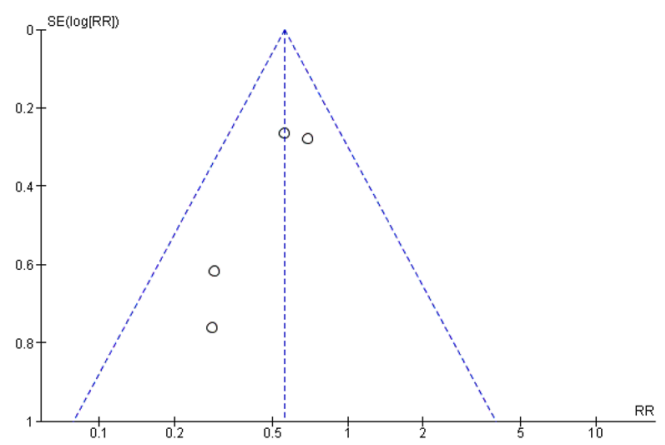

Fig. 8 Funnel plot showing the assessment of publication bias

Another limitation of note is the temporal spread of the studies as perioperative practice has undergone many changes over the past 16 years and may well have created differences between the earliest study ${ }^{12}$ to the most recent study. ${ }^{33}$ Therefore 'standard care' received by patients in the control arm was probably not standardised between studies. The same undoubtedly applies for postoperative management algorithms where there has been a shift towards early resumption of oral intake, optimisation of fluid management, the selective use of anaesthesia, and the use of medications to reduce POI such as Alvimopan. Furthermore, none of the studies utilising ERAS pathways included an assessment of compliance with their ERAS standards. This is of significant relevance due to the positive correlation between compliance with standards and clinical outcomes. Additionally, given that some studies had not incorporated ERAS principles, the impact of chewing gum in a firm setting of enhanced recovery remains undefined.

Finally, one might argue against the effectiveness of chewing gum in reducing the occurrence of POI, as no significant reduction in the length of hospital stay was demonstrated. However, as our findings revealed that neither the time to passage of flatus nor the time to defecation was reduced by more than a day with the use of sham feeding with chewing gum, we did not expect a significant reduction in length of stay. Moreover, the various non-clinical factors which affect hospital discharge across different centres, in addition to nonstandardised ERAS protocols would suggest that length of hospital stay may not be an accurate outcome indicator for measuring the effectiveness of chewing gum at reducing POI. ${ }^{36-38}$ It is therefore difficult to state that because length of hospital stay was unimproved, the impact of chewing gum is without merit, as our meta-analysis does demonstrate a significant reduction in the reporting of ileus, time to passage of flatus and time to passage of stool.

\section{Comparison with Other Studies}

Our findings follow a similar trend to previous systematic reviews by Fitzgerald and Sua who found significant
Table 2 Definitions of postoperative ileus by study

\begin{tabular}{|c|c|}
\hline Study & Definition of postoperative ileus used in study \\
\hline Asao et al. $(2002)^{12}$ & None \\
\hline Atkinson et al. $(2016)^{21}$ & $\begin{array}{l}\text { Clinical state characterised by nausea, vomiting, abdominal distension and an } \\
\text { inability to pass stools/flatus }\end{array}$ \\
\hline Bonventre et al. $(2014)^{16}$ & $\begin{array}{l}\text { A transient cessation of coordinated bowel motility after surgical intervention, } \\
\text { which prevents effective transit of intestinal contents and/or tolerance of intake }\end{array}$ \\
\hline Crainic et al. $(2009)^{22}$ & None \\
\hline Forrester et al. $(2014)^{23}$ & Cessation of bowel function following surgery that lasts 3 days or longer \\
\hline Hirayama et al. $(2006)^{24}$ & None \\
\hline $\begin{array}{l}\text { Kobayashi et al. } \\
(2015)^{25}\end{array}$ & None \\
\hline Lim et al. $(2013)^{26}$ & None \\
\hline Matros et al. $(2006)^{27}$ & $\begin{array}{l}\text { Transient cessation of luminal transit after laparotomy or laparoscopy because of } \\
\text { inhibition of intestinal motility }\end{array}$ \\
\hline Quah et al. $(2005)^{28}$ & The delayed return of coordinated intestinal motility following abdominal surgery \\
\hline Shum et al. $(2016)^{29}$ & None \\
\hline Schuster et al. $(2006)^{10}$ & None \\
\hline Topcu et al. $(2016)^{30}$ & None \\
\hline $\begin{array}{l}\text { Van den Heijkant et al. } \\
\quad(2015)^{31}\end{array}$ & $\begin{array}{l}\text { A lack of passage of flatus or stool and intolerance to oral intake for at least } 24 \mathrm{~h} \text { on } \\
\text { day } 5 \text { postoperatively }\end{array}$ \\
\hline $\begin{array}{l}\text { Vergara-Fernandez et al. } \\
\quad(2016)^{32}\end{array}$ & $\begin{array}{l}\text { Absence of adequate bowel function on postoperative day } 5 \text {, or the need for the } \\
\text { insertion of a nasogastric tube because of abdominal distension, nausea and } \\
\text { emesis after having started a liquid diet, in the absence of mechanical obstruction }\end{array}$ \\
\hline Yang et al. $(2018)^{33}$ & $\begin{array}{l}\text { Two or more episodes of nausea/vomiting, inability to tolerate oral diet over } 24 \mathrm{~h} \text {, } \\
\text { absence of flatus over } 24 \mathrm{~h} \text {, distension and radiologic confirmation on or after } \\
\text { postoperative day } 4 \text { without prior resolution }\end{array}$ \\
\hline
\end{tabular}


Table 3 Summary of incidence of postoperative ileus, time to flatus and time to defecation across all studies

\begin{tabular}{|c|c|c|c|c|c|c|c|c|c|c|c|c|}
\hline \multirow[t]{3}{*}{ Study } & \multicolumn{4}{|c|}{ Postoperative ileus incidence $(n)$} & \multicolumn{4}{|c|}{ Time to flatus (days) } & \multicolumn{4}{|c|}{ Time to defecation (days) } \\
\hline & \multicolumn{2}{|c|}{ Intervention } & \multicolumn{2}{|l|}{ Control } & \multicolumn{2}{|l|}{ Intervention } & \multicolumn{2}{|l|}{ Control } & \multicolumn{2}{|l|}{ Intervention } & \multicolumn{2}{|l|}{ Control } \\
\hline & $n(\%)$ & Total & $n(\%)$ & Total & Mean \pm SD & Total & Mean \pm SD & Total & Mean \pm SD & Total & Mean \pm SD & Total \\
\hline Asao et al. $(2002)^{12}$ & - & - & - & - & - & - & - & - & - & - & - & - \\
\hline Atkinson et al. $(2016)^{21}$ & $19(10)$ & 199 & $28(14)$ & 202 & - & - & - & - & $2 \pm 1.50$ & 159 & $2.67 \pm 2.25$ & 151 \\
\hline Bonventre et al. $(2014)^{16}$ & - & - & - & - & - & - & - & - & - & - & - & - \\
\hline Crainic et al. $(2009)^{22}$ & - & - & - & - & - & - & - & - & - & - & - & - \\
\hline Forrester et al. $(2014)^{23}$ & - & - & - & - & $2.98 \pm 3.16$ & 13 & $3.42 \pm 2.18$ & 17 & $5.21 \pm 4.62$ & 9 & $3.06 \pm 2.63$ & 15 \\
\hline Hirayama et al. $(2006)^{24}$ & - & - & - & - & - & - & - & - & - & - & - & - \\
\hline Kobayashi et al. $(2015)^{25}$ & - & - & - & - & $2.21 \pm 0.08$ & 21 & $2.04 \pm 1.08$ & 22 & $3.92 \pm 1.83$ & 21 & $4.54 \pm 1.46$ & 22 \\
\hline Lim et al. $(2013)^{26}$ & - & - & - & - & $1.78 \pm 0.16$ & 77 & $2.12 \pm 0.16$ & 80 & $3.74 \pm 0.25$ & 77 & $4.11 \pm 0.29$ & 80 \\
\hline Matros et al. $(2006)^{27}$ & - & - & - & - & $2.63 \pm 0.76$ & 22 & $2.9 \pm 0.92$ & 21 & $3.5 \pm 1.12$ & 22 & $3.68 \pm 1.29$ & 21 \\
\hline Quah et al. $(2005)^{28}$ & - & - & - & - & $2.4 \pm 1$ & 19 & $2.7 \pm 1$ & 19 & $3.2 \pm 1.5$ & 19 & $3.9 \pm 1.5$ & 19 \\
\hline Shum et al. $(2016)^{29}$ & - & - & - & - & $1.57 \pm 2.63$ & 41 & $2.57 \pm 4.39$ & 41 & $1.46 \pm 2.43$ & 41 & $2.85 \pm 4.58$ & 41 \\
\hline Schuster et al. $(2006)^{10}$ & - & - & - & - & - & - & - & - & - & - & - & - \\
\hline Topcu et al. $(2016)^{30}$ & - & - & - & - & - & - & - & - & - & - & - & - \\
\hline Van den Heijkant et al. $(2015)^{31}$ & $14(27)$ & 52 & $29(48)$ & 60 & - & - & - & - & - & - & - & - \\
\hline Vergara-Fernandez et al. $(2016)^{32}$ & $2(6)$ & 32 & $7(22)$ & 32 & - & - & - & - & - & - & - & - \\
\hline Yang et al. $(2018)^{33}$ & $3(7)$ & 43 & $11(24)$ & 46 & $1.76 \pm 0.14$ & 43 & $2.05 \pm 0.06$ & 46 & $2.75 \pm 0.1$ & 43 & $3.27 \pm 0.07$ & 46 \\
\hline
\end{tabular}

- data not available

reduction in time to passage of flatus and time to bowel movement, and no significant difference in length of hospital stay and complication rates. ${ }^{9}, 39$ Since we would not expect any harm from chewing gum, it was not surprising to find no significant association with complication rates, reaffirming the safety of chewing gum use postoperatively.

Nonetheless, our findings diverge from other reviews with regard to length of stay. A 2015 Cochrane review ${ }^{13}$ demonstrated a significant reduction in time to first flatus, time to bowel movement and length of hospital stay in patients who had chewing gum compared to control groups, echoed by two other reviews. ${ }^{8,40}$ However, it should be noted that this review included studies with paediatric patients, and patients undergoing gynaecological procedures, which might have affected the results.

\section{Health Policy Implications}

Taking into account that POI is the most frequent complication after gastrointestinal surgery, any incremental benefit in reducing the duration of symptoms is likely to be beneficial overall. Chewing gum as an alternative to early enteral feeding is proven to be a safe and effective intervention. Administration of chewing gum on the first postoperative day could prevent POI and the morbidity associated with delayed gut motility.

\section{Conclusion}

This meta-analysis of ten moderate to high quality RCTs provides evidence of the benefit of using sham feeding with chewing gum to reduce the incidence of POI in patients undergoing colorectal surgery. While the length of hospital stay is unimproved, there is clear reduction in the time to passage of flatus and time to defecation. Recognising that POI has a multifactorial underlying pathophysiology, chewing gum is unlikely to be the sole answer to the complex problem of POI in patients undergoing colorectal surgery. However, given the low sideeffect profile, wide availability and patient acceptance of postoperative chewing gum use in addition to its potential benefit of reducing POI suggests it should be routinely considered as part of existing ERAS protocols.

Author Contributions All named authors have been involved in the planning, conduct and reporting of the study, and accept full responsibility for the decision to publish. The corresponding author attests that all listed authors meet authorship criteria and that no others meeting the criteria have been omitted.

\section{Compliance with Ethical Standards}

Conflict of Interest The authors declare that they have no conflict of interest. 
Open Access This article is licensed under a Creative Commons Attribution 4.0 International License, which permits use, sharing, adaptation, distribution and reproduction in any medium or format, as long as you give appropriate credit to the original author(s) and the source, provide a link to the Creative Commons licence, and indicate if changes were made. The images or other third party material in this article are included in the article's Creative Commons licence, unless indicated otherwise in a credit line to the material. If material is not included in the article's Creative Commons licence and your intended use is not permitted by statutory regulation or exceeds the permitted use, you will need to obtain permission directly from the copyright holder. To view a copy of this licence, visit http://creativecommons.org/licenses/by/4.0/.

\section{References}

1. Vather R, Trivedi S, Bissett I. Defining Postoperative Ileus: Results of a Systematic Review and Global Survey. J Gastrointest Surg. 2013;17(5):962-972. https://doi.org/10.1007/s11605-013-2148-y

2. Chapuis PH, Bokey L, Keshava A, et al. Risk factors for prolonged ileus after resection of colorectal cancer: An observational study of 2400 consecutive patients. Ann Surg. 2013;257(5):909-915. https:// doi.org/10.1097/SLA.0b013e318268a693

3. Asgeirsson, Theodor; El-Badawi, Khaled; Mahmood, Ali; Barletta, Jeffrey; Luchtefeld, Martin; Senagore AJ. Postoperative Ileus: It Costs More Than You Expect. J Am Coll Surg. 2010;210(2):228231.

4. Goldstein JL, Matuszewski KA, Delaney CP, et al. Inpatient Economic Burden of Postoperative Ileus Associated with Abdominal Surgery in the United States. 2007;32(2).

5. Venara A, Neunlist M, Slim K, et al. Postoperative ileus: Pathophysiology, incidence, and prevention. J Visc Surg. 2016;153(6):439-446. https://doi.org/10.1016/j.jviscsurg.2016.08. 010

6. Gero D, Gié O, Hübner M, Demartines N, Hahnloser D. Postoperative ileus: in search of an international consensus on definition, diagnosis, and treatment. Langenbeck's Arch Surg. 2017;402(1):149-158. https://doi.org/10.1007/s00423-016-1485-1

7. ERAS. http://erassociety.org/.

8. Noble EJ, Harris R, Hosie KB, Thomas S, Lewis SJ. Gum Chewing Reduces Postoperative Ileus? A Systematic Review and MetaAnalysis. Int J Surg. 2009;7(2):100-105. https://doi.org/10.1016/j. ijsu.2009.01.006

9. Su'a BU, Pollock TT, Lemanu DP, MacCormick AD, Connolly AB, Hill AG. Chewing gum and postoperative ileus in adults: A systematic literature review and meta-analysis. Int J Surg. 2015;14(15):49-55. https://doi.org/10.1016/j.ijsu.2014.12.032

10. Schuster R, Grewal N, Greaney GC, Waxman K. Gum chewing reduces ileus after elective open sigmoid colectomy. Arch Surg. 2006;141(2):174-176. https://doi.org/10.1001/archsurg.141.2.174

11. Lassen Kristoffer; Kjave Jorn FT et al. Allowing Normal Food at Will After Major Upper Gastrointestinal Surgery Does Not Increase Morbidity: A Randomized Multicenter Trial. Ann Surg. 2008;247(Issue 5):721-729. https://doi.org/10.1097/SLA. 0b013e31815cca68

12. Asao T, Kuwano H, Nakamura Jichi, Morinaga N, Hirayama I, Ide M. Gum chewing enhances early recovery from postoperative ileus after laparoscopic colectomy. J Am Coll Surg. 2002;195(1):30-32. https://doi.org/10.1016/S1072-7515(02)01179-1

13. Short, Vanessa; Herbert, Georgia; Perry R. Chewing gum for postoperative recovery of gastrointestinal function. Cochrane Database Syst Rev. 2015;(February):1-221. https://www.cochranelibrary. com/cdsr/doi/10.1002/14651858.CD006506.pub3/full
14. SIGN search-filters-randomised-controlled-trials.

15. Moher D, Liberati A, Tetzlaff J, Altman DG, The PRISMA Group. PRISMA 2009 Flow Diagram. PLoS Med. 2009;6(7):e1000097. https://doi.org/10.1371/journal.pmed1000097

16. Bonventre S, Inviati A, Di Paola V, et al. Evaluating the efficacy of current treatments for reducing postoperative ileus: A randomized clinical trial in a single center. Minerva Chir. 2014;69(1):47-55.

17. Hozo SP, Djulbegovic B, Hozo I. Estimating the mean and variance from the median, range, and the size of a sample. BMC Med Res Methodol. 2005;5:1-14. https://doi.org/10.1186/1471-2288-5-13

18. Wan X, Wang W, Liu J, Tong T. Estimating the sample mean and standard deviation from the sample size, median, range and/or interquartile range. 2014:1-13.

19. Jadad AR, Moore RA, Carroll D, et al. Assessing the Quality of Reports of Ramdomized Clinical Trials: Is Blinding Necessary? 1996;17(January 1996):1-12. https://doi.org/10.1016/01972456(95)00134-4

20. The Cochrane Collaboration. RevMan 5.3. 2017. https:// community.cochrane.org/help/tools-and-software/revman-5/ revman-5-download.

21. Atkinson C, Penfold CM, Ness AR, et al. Randomized clinical trial of postoperative chewing gum versus standard care after colorectal resection. Br J Surg. 2016;103(8):962-970. https://doi.org/10. 1002/bjs. 10194

22. Crainic C, Erickson K, Gardner J, et al. Comparison of methods to facilitate postoperative bowel function. Medsurg Nurs. 2009;18(4): 235-238. http://www.ncbi.nlm.nih.gov/pubmed/20552850.

23. Forrester DA (Tony), Doyle-Munoz J, McTigue T, D Andrea S, Natale-Ryan A. The Efficacy of Gum Chewing in Reducing Postoperative Ileus. J Wound, Ostomy Cont Nurs. 2014;41(3): 227-232. https://doi.org/10.1097/WON.0000000000000019

24. Hirayama I, Suzuki M, Ide M, Asao T, Kuwano H. Gum-chewing stimulates bowel motility after surgery for colorectal cancer. Hepatogastroenterology. 2006;53(68):206-208.

25. Kobayashi T, Masaki T, Kogawa K, Matsuoka H, Sugiyama M. Efficacy of Gum Chewing on Bowel Movement After Open Colectomy for Left-Sided Colorectal Cancer: A Randomized Clinical Trial. Dis Colon Rectum. 2015;58(11):1058-1063. https://doi.org/10.1097/DCR.0000000000000452

26. Lim P, Morris OJ, Nolan G, Moore S, Draganic B, Smith SR. Sham feeding with chewing gum after elective colorectal resectional surgery: A randomized clinical trial. Ann Surg. 2013;257(6):10161024. https://doi.org/10.1097/SLA.0b013e318286504a

27. Matros E, Rocha F, Zinner M, et al. Does Gum Chewing Ameliorate Postoperative Ileus? Results of a Prospective, Randomized, Placebo-Controlled TrialJ Am Coll Surg. 2006;202(5):773-778. https://doi.org/10.1016/j.jamcollsurg.2006. 02.009

28. Quah HM, Samad A, Neathey AJ, Hay DJ, Maw A. Does gum chewing reduce postoperative ileus following open colectomy for left-sided colon and rectal cancer? - A prospective randomized controlled trial. Color Dis. 2006;8(1):64-70. https://doi.org/10.1111/j. 1463-1318.2005.00884.x

29. Shum NF, Choi HK, Mak JCK, Foo DCC, Li WC, Law WL. Randomized clinical trial of chewing gum after laparoscopic colorectal resection. Br J Surg. 2016;103(11):1447-1452. https://doi. org/10.1002/bjs. 10277

30. Topcu SY, Oztekin SD. Effect of gum chewing on reducing postoperative ileus and recovery after colorectal surgery: A randomised controlled trial. Complement Ther Clin Pract. 2016;23:21-25. https://doi.org/10.1016/j.ctcp.2016.02.001

31. Van Den Heijkant TC, Costes LMM, Van Der Lee DGC, et al. Randomized clinical trial of the effect of gum chewing on postoperative ileus and inflammation in colorectal surgery. $\mathrm{Br} J$ Surg. 2015;102(3):202-211. https://doi.org/10.1002/bjs.9691 
32. Vergara-Fernandez O, Gonzalez-Vargas AP, Castellanos-Juarez JC, Salgado-Nesme N, Sanchez-Garcia Ramos E. Usefulness of Gum Chewing to Decrease Postoperative Ileus in Colorectal Surgery with Primary Anastomosis: A Randomized Controlled Trial. Rev Invest Clin. 2016;68(6):314-318.

33. Yang P, Long WJ, Li W. Chewing Xylitol Gum could Accelerate Bowel motility Recovery after Elective Open Proctectomy for Rectal Cancer. 2018:53-58. https://doi.org/10.24875/RIC. 18002428

34. Aravani A, Samy EF, Thomas JD, Quirke P. A retrospective observational study of length of stay in hospital after colorectal cancer surgery in England (1998-2010). 2019;95(47):1-13. https://doi. org/10.1097/MD.0000000000005064

35. Shepperd S, Parkes J, Jjm M, et al. Discharge planning from hospital to home (Review). 2009;(1):10-12. https://doi.org/10.1002/ 14651858.CD000313.pub2.Copyright

36. Brasel K, Lim H, Nirula R WJ. Length of stay - An Appropriate Quality Measure? Arch Surg. 2007;142:461-466. https://doi.org/ 10.1176/ps.38.2.201

37. Lingsma HF, Bottle A, Middleton S, Kievit J, Steyerberg EW, Marang-Van De Mheen PJ. Evaluation of hospital outcomes: The relation between length-of-stay, readmission, and mortality in a large international administrative database. BMC Health Serv Res. 2018;18(1):1-10. https://doi.org/10.1186/s12913-018-2916-1

38. Chapman SJ, Thorpe G, et al. Systematic review of definitions and outcome measures for return of bowel function after gastrointestinal surgery. BJS Open. 2019;3(1):1-10. https://doi.org/10.1002/bjs5. 102

39. Fitzgerald JEF, Ahmed I. Systematic review and meta-analysis of Chewing-gum therapy in the reduction of postoperative paralytic ileus following gastrointestinal surgery. World J Surg. 2009;33(12): 2557-2566. https://doi.org/10.1007/s00268-009-0104-5

40. Zhang, Hui; Deng, Yong-Hong; Shuai, Ting; Song G-M. Chewing gum for postoperative ileus after colorectal surgery: A systematic review of overlapping meta-analyses. 2017;4(2):92-104. https:// doi.org/10.1016/j.ecns.2009.08.002.https

Publisher's Note Springer Nature remains neutral with regard to jurisdictional claims in published maps and institutional affiliations. 